\title{
Pistas diretas no tratamento de bruxismo infantil: relato de caso
}

Direct tracks in the treatment of child brussels: case report

Pistas directas en el tratamiento de la bruja infantil: informe de caso

Gíssia Santana CARMO ${ }^{1}$

Joyce Ferreira Nobre CAVALCANTE ${ }^{1}$

José Henrique de Araújo $\mathbf{C R U Z}^{\mathbf{2}}$

Fatima Roneiva Alves FONSECA ${ }^{3}$

Maria Carolina Bandeira MACENA ${ }^{3}$

${ }^{1}$ Graduação em Odontologia, Unidade Acadêmica de Ciências Biológicas, Universidade Federal de Campina Grande (UFCG), 58708-110 Patos - PB, Brasil

${ }^{2}$ Cirurgião-Dentísta Curso de Odontologia, Unidade Acadêmica de Ciências Biológicas, Universidade Federal de Campina Grande (UFCG), 58708-110 Patos - PB, Brasil

${ }^{3}$ Professora Doutora, Curso de Odontologia, Unidade Acadêmica de Ciências Biológicas, Universidade Federal de Campina Grande (UFCG), 58708-110 Patos - PB, Brasil

\section{Resumo}

Introdução: O bruxismo infantil é um ato inconsciente que pode estar atrelado a fatores hereditários e psicológicos, uma desordem funcional, que é caracterizada pelo típico ranger de dentes, com forças oclusais extremamente elevadas quando comparadas ao normal que são capazes de gerar, a médio e longo prazo, grandes consequências. Objetivo: Relatar um caso clínico de confecção e instalação de pistas diretas planas em paciente infantil, realizado na Clínica Escola de Odontologia da Universidade Federal de Campina Grande (UFCG), com intuito de considerar a eficácia do tratamento. Relato do caso: Este estudo apresenta o caso de um paciente infantil, 10 anos, que procurou atendimento odontológico com queixa de bruxismo. Através de anamnese e exame clínico, foi possível observar a presença dessa disfunção. O tratamento proposto foi instalação de pistas diretas planas em molares inferiores. O procedimento foi iniciado a partir da moldagem anatômica com moldeiras infantis 2 e alginato tipo II, seguido de vazamento do molde em gesso. Com modelos vazados, utilizou-se resina composta e espátula de resina para confecção das pistas, foi realizado polimento das mesmas adaptadas no modelo com pedra pomes. A instalação, foi precedida por profilaxia, ataque ácido e enxague, inserção de adesivo e fotopolimerização. As pistas foram adaptadas individualmente com auxílio de resina flow e fotoativação nos elementos $74,75,84,85$. Conclusão: 0 tratamento escolhido baseou-se em técnica bastante prática e reversível, que demonstrou grande êxito e proporcionou relaxamento muscular com consequente ausência de sintomatologia.

Descritores: Bruxismo; Ortodontia; Odontopediatria; Estresse.

\section{Abstract}

Introduction: Infantile bruxism is an unconscious act that may be linked to hereditary and psychological factors, a functional disorder, which is characterized by the typical gnashing of teeth, with extremely high occlusal forces when compared to normal that are capable of generating, in the medium and long term, great consequences. Objective: To report a clinical case of preparation and installation of flat direct clues in a child patient, carried out at the School of Dentistry Clinic of the Federal University of Campina Grande (UFCG), in order to consider the efficacy of the treatment. Case report: This study presents the case of a 10-year-old infant patient who sought dental care complaining of bruxism. Through anamnesis and clinical examination, it was possible to observe the presence of this dysfunction. The proposed treatment was installation of flat direct clues in lower molars. The procedure was initiated from anatomical molding with infant trays 2 and alginate type II, followed by leakage of the cast in plaster. With leaked models, composite resin and resin spatula were used to make the tracks, polishing them adapted in the pumice model. The installation was preceded by prophylaxis, acid attack and rinsing, adhesive insertion and light curing. The tracks were individually adapted with the aid of flow resin and photoactivation in elements $74,75,84,85$. Conclusion: The treatment chosen was based on a very practical and reversible technique, which demonstrated great success and provided muscle relaxation with consequent absence of symptomatology.

Descriptors: Bruxism; Orthodontics; Pediatric Dentistry; Stress.

\section{Resumen}

Introducción: el bruxismo infantil es un acto inconsciente que puede estar relacionado con factores hereditarios y psicológicos, un trastorno funcional, que se caracteriza por el típico rechinar de dientes, con fuerzas oclusales extremadamente altas en comparación con los normales que son capaces de generar, a mediano y largo plazo. a largo plazo, grandes consecuencias. Objetivo: informar un caso clínico de confección e instalación de pistas planas directas en un paciente infantil, realizado en la Escuela Clínica de Odontología de la Universidad Federal de Campina Grande (UFCG), para considerar la efectividad del tratamiento. Informe del caso: Este estudio presenta el caso de un paciente infantil de 10 años que buscó atención dental quejándose de bruxismo. A través de la anamnesis y el examen clínico, fue posible observar la presencia de esta disfunción. El tratamiento propuesto fue la instalación de pistas planas directas en los molares mandibulares. El procedimiento se inició a partir de la impresión anatómica con bandejas infantiles 2 y alginato tipo II, seguido de la fundición del yeso. Con los modelos huecos, se utilizaron resina compuesta y espátula de resina para hacer las pistas, puliéndolas adaptadas en el modelo con piedra pómez. La instalación fue precedida por profilaxis, ataque con ácido y enjuague, inserción de adhesivo y fotopolimerización. Las pistas se adaptaron individualmente con la ayuda de la resina de flujo y la fotoactivación en los elementos 74, 75, 84, 85. Conclusión: El tratamiento elegido se basó en una técnica muy práctica y reversible, que demostró ser altamente exitosa y proporcionó relajación muscular con la consiguiente ausencia de síntomas.

Descriptores: Bruxismo; Ortodoncia; Odontología Pediátrica; Estrés.

INTRODUÇÃO

O estresse é caracterizado por
apresentar reações que irritam, amedrontam e anseiam, podendo ser causado por situações felizes ou tristes e que é considerado prejudicial dependendo da frequência e se houver predisposição para isso. $O$ estresse pode ser observado com frequência na classe infantil, que se assemelha ao adulto em grandes situações que podem envolver bloqueios em interação, socialização e situações de grande ansiedade, que podem gerar grandes consequências quando excessivo ${ }^{1-3}$. 
Em 1907, o bruxismo foi descrito na literatura científica; conhecido pelo típico ranger de dentes. As forças oclusais realizadas pelo indivíduo são extremamente elevadas quando comparadas ao normal. A partir dessa disfunção, variam-se as consequências de acordo com a intensidade de cada paciente, desde a ocorrência localizada ou generalizada de facetas de desgate, hipersensibilidade térmica, necrose pulpar, dores musculares e articulares, perda de dimensão vertical, além de disfunções temporomandibulares ${ }^{4}$.

A etiologia do bruxismo está diretamente relacionada com fatores psicológicos, sistêmicos, hereditários e locais ${ }^{4,5}$. Além de deficiências sistêmicas, as alergias, parasitoses intestinais e desordens endócrinas também vêm sido correlacionadas como fatores causais do bruxismo ${ }^{6}$.

O bruxismo também afeta o público infantil, e é caracterizado como uma atividade parafuncional, com a presença de contrações musculares, onde os mecanismos de proteção neuromusculares não estão presentes e acontecem quando o indivíduo está no nível subconsciente, no estágio REM (Rapid Eye Movement) na maioria das vezes ${ }^{7}$.

O período da infância é um precursor para a ocorrência de disfunções temporomandibulares e consequentes danos ao sistema estomatognático. $\mathrm{O}$ atendimento odontológico e observacional por parte dos responsáveis é de grande importância, sua carência pode acarretar consequências severas na musculatura facial e na cavidade oral ${ }^{8-11}$.

Após avaliação clínica, o bruxismo pode estar relacionado com níveis de desgaste nas superfícies incisais e oclusais dos dentes, quase sempre acompanhados de incômodos articulares e musculares ${ }^{12,13}$.

O tratamento indicado evidencia a importância multidisciplinar, interligando a odontologia, psicologia e medicina. O tratamento pode envolver terapias comportamentais, visando reduzir o estresse, melhorar os níveis de ansiedade e melhora do sono ${ }^{14,15}$. $\mathrm{Na}$ parte odontológica, a terapia compreende procedimentos restauradores, ortodônticos, confecção de placas, instalação de pistas Planas, de acordo com a situação clínica e queixa principal do paciente ${ }^{16-18}$.

Este estudo tem por objetivo relatar um caso clínicio de um paciente infantil, sexo masculino, 10 anos de idade, que apresentava bruxismo e compareceu à Clínica Escola de Odontologia da Universidade Federal de Campina Grande (UFCG), relatando ranger os dentes no período da noite.
CASO CLÍNICO

Paciente do sexo masculino,

melanoderma, 10 anos de idade, residente na cidade de Patos Paraíba, compareceu à Clínica Escola de Odontologia da Universidade Federal de Campina Grande (UFCG) na especialidade de Odontopediatria e Ortodontia. A criança relatava frequentes dores de cabeça e cansaço na musculatura da face, e a mãe queixava-se da presença do ranger dos dentes do filho durante à noite, além de noites mal dormidas com várias interrupções. Após anamnese e avaliação clínica foi possível observar a presença de atividade parafuncional, causando desgaste na incisal de alguns elementos dentários, além de uma expressiva mordida profunda conforme as Figura 1 e 2.

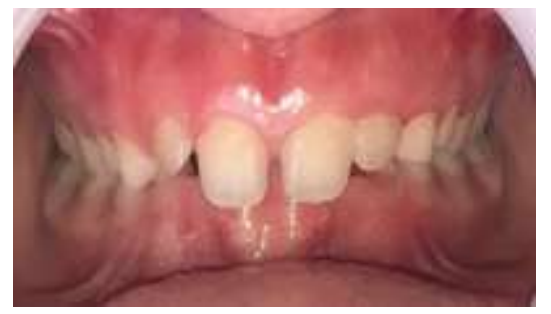

Figura 1: Expressiva mordida profunda.

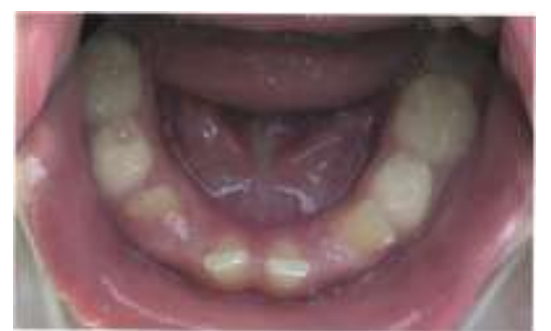

Figura 2: Desgastes incisais nas distais dos elementos 31 e 41

O tratamento de escolha foi a utilização de Pistas Diretas Planas (PDP) nos molares 74, $75,84,85$, os primeiros molares permanentes não recebem as PDP para que seja permitida sua erupção passiva, ajustando a dimensão vertical de oclusão e corrigindo, ao final do tratamento, a mordida profunda. Sendo uma técnica de grande praticidade e resultados positivos que permite auxiliar o tratamento do bruxismo, e que é considerada uma terapia ortopédica que reposiciona a mandíbula com melhorias em más funções músculoesqueléticas.

Iniciou-se a partir da moldagem anatômica com utilização de moldeiras infantis tamanho 2 (Maquira, Brasil) e alginato tipo II (Dencrigel, Brasil) (Figura 3) e posterior vazamento do molde em gesso pedra tipo III (Asfer, Brasil) para obtenção do modelo de gesso (Figura 4). Com os modelos de gesso vazados, foi utilizado resina composta cor dA1 Opallis LAB (FGM, Brasil), com auxílio de uma espátula de resina em titânio $n^{\circ} 05$ (Millennium, Brasil), e fotopolimerizador (Gnatus, Brasil). 


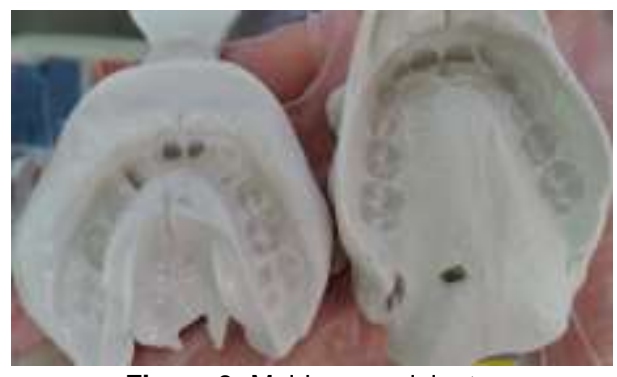

Figura 3: Moldes em alginato.

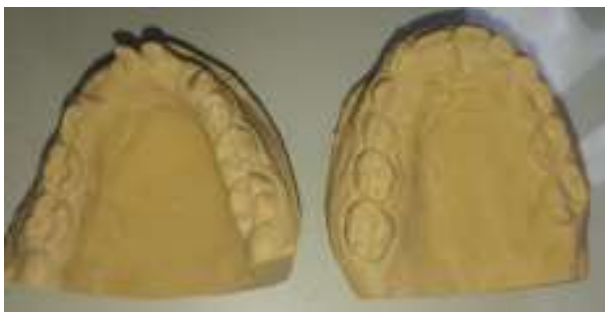

Figura 4: Modelos de gesso.

As pistas foram adaptadas no modelo de gesso por meio de cera 07 (Newwax 7, Brasil), e polidas com pedra pomes (Biodinâmica, Brasil) com auxílio de peça reta (NSK, Japão) e disco de feltro adaptado na mesma (Figura 5).

Após confeccionadas, numa segunda consulta odontológica, foi realizada profilaxia em todos os elementos (Figura 6), condicionamento ácido do esmalte com ácido fosfórico $37 \%$ por 30 segundos (Attaque Gel, Biodinâmica, Brasil) (Figura 7), lavagem e secagem, seguida de inserção e fotopolimerização de adesivo Prime Bond 21 (Dentsplay, Brasil).

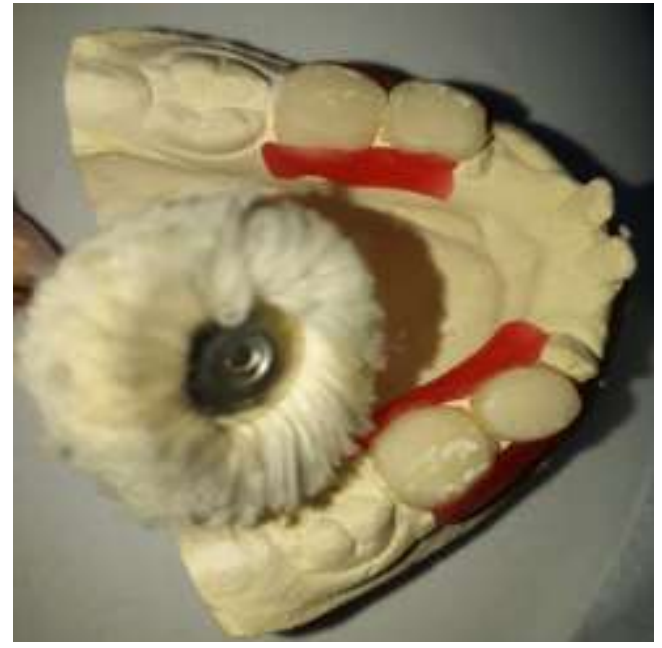

Figura 5: Polimento pré-instalação.

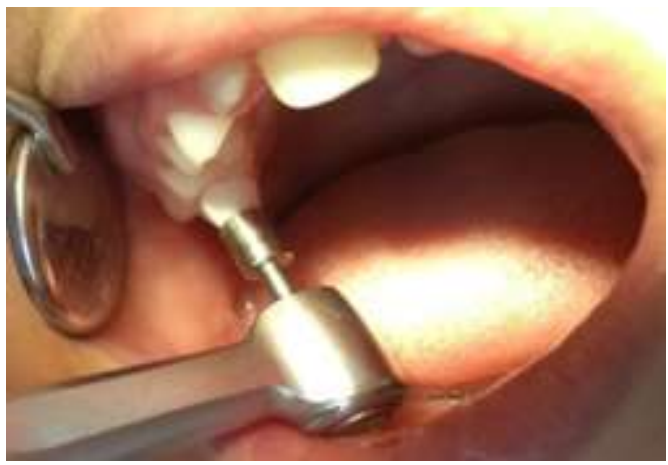

Figura 6: Profilaxia de todos os elementos dentários.

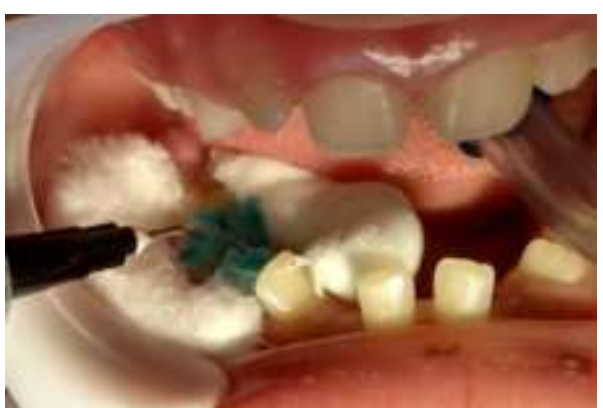

Figura 7: Condicionamento ácido do esmalte.

Na sequência, procedeu-se inserção de resina master flow (Biodinâmica, Brasil) individualmente nas pistas (Figura 8), seguido de fotopolimerização nos elementos. Foi utilizado fio dental (Oral B, Brasil) entre os elementos onde foram instaladas as PDP para verificar os contatos interproximais e remover qualquer excesso de adesivo ou resina (Figura 9).

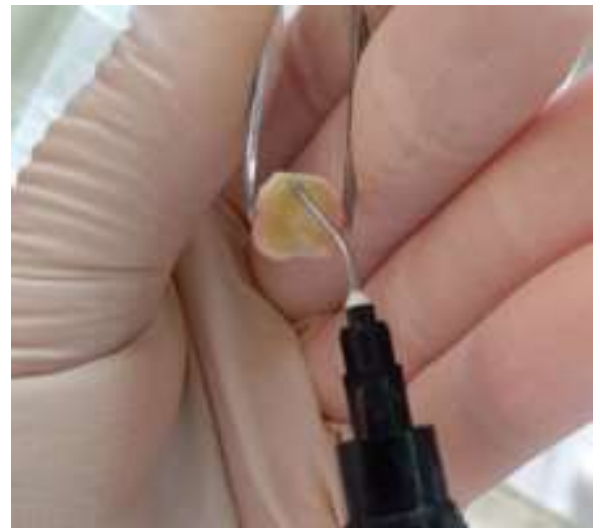

Figura 8: Aplicação de resina master flow individualmente nas pistas.

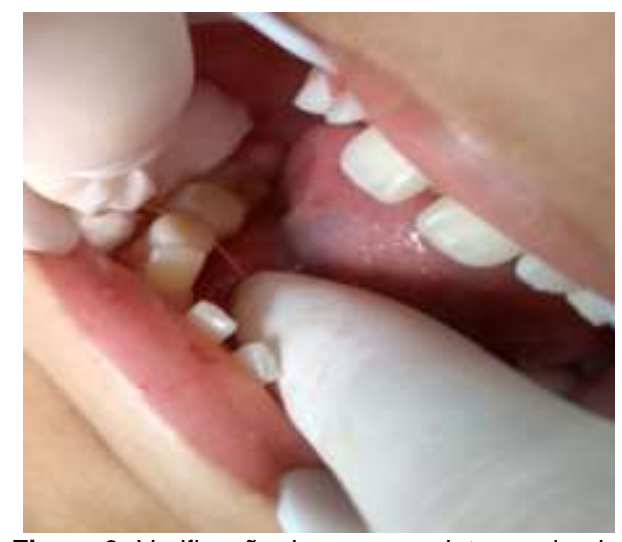

Figura 9: Verificação dos espaços interproximais

Adaptadas aos dentes (Figura 10) e verificados os ajustes oclusais com papel carbono (Figura 11), foi realizado acabamento com pontas diamantadas 2135ff e 3195ff (Microdont, Brasil) (Figura 12), as pistas planas diretas possibilitaram um aumento significativo da Dimensão Vertical de Oclusão (DVO) (Figura 13). O paciente foi acompanhado por um período de tempo de 6 meses, período em que observou-se estagnação dos desgastes incisais e relato da mãe de ausência de rangidos durante a noite, melhora no sono 
noturno, redução das dores de cabeça e diminuição do cansaço facial. Após o período de tempo determinado para o tratamento do bruxismo, as PDP foram removidas com auxílio de pontas diamantadas esféricas.

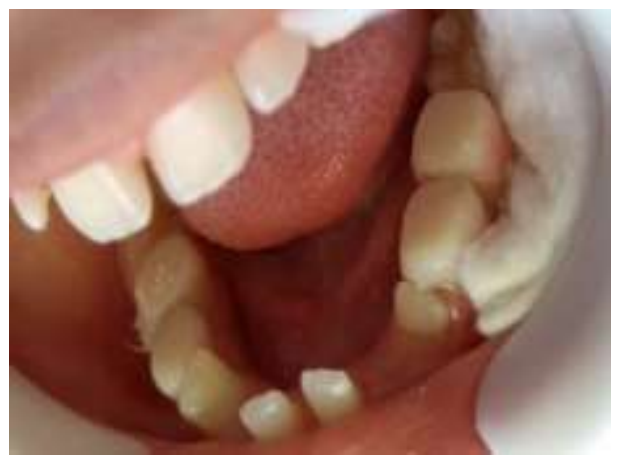

Figura 10: Pistas instaladas

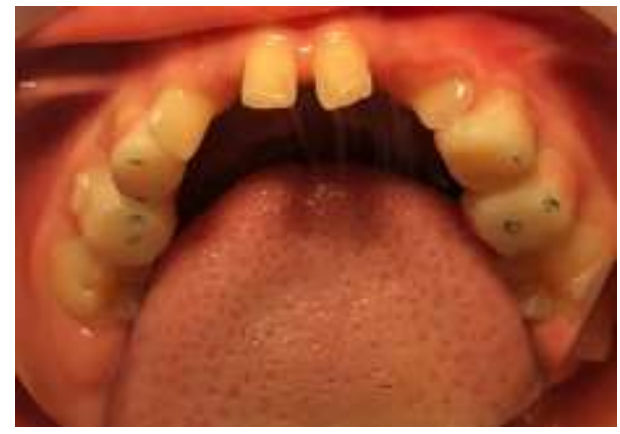

Figura 11: Verificação oclusal com papel carbono.

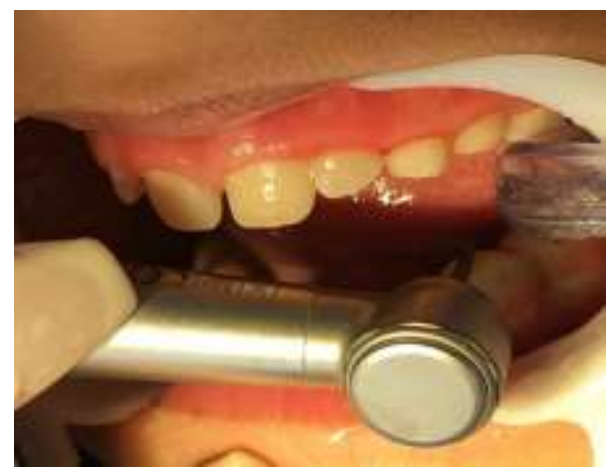

Figura 12: Ajuste oclusal com brocas 3195FF e 2135FF.

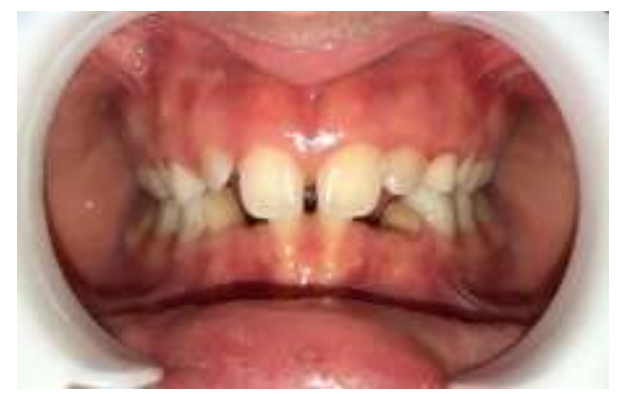

Figura 13: Aumento significativo da DVO após instalação de PDP DISCUSSÃO

Estudos que corroboram entre si como os de Diniz et al. ${ }^{19}$, Gonçalves et al. ${ }^{20}$, Macedo ${ }^{21}$ consideram o bruxismo como uma atividade parafuncional presente no sistema mastigatório e não uma doença, e a caracterizam por ser de origem multifatorial, além disso, a classificam sua origem, podendo ser primário ou secundário; primário aquele que não possui causa aparente, e o secundário por estar relacionado a algum fator extrínseco de desordem neurológica ou psiquiátrica.

Para Masuko et al. ${ }^{22}$, além do ranger de dentes, afirmam que normalmente as crianças com essa sintomatologia apresentam episódios recorrentes de cefaleia. Paiva et al. $^{23}$ complementa ainda esta linha de raciocínio evidenciando que a presença dessa atividade parafuncional compartilhava de dores de cabeça como uma morbidade associada ao problema.

De acordo com vários autores, inclusive Ahmad $^{12}$, aconselham a utilização do ajuste oclusal com o intuito de remover os pontos de contatos prematuros, e ainda complementam a ideia de que em casos mais graves deve se realizar de forma cuidadosa procedimentos que visem o aumento da DVO.

Corroborando com o estudo de Ahmad ${ }^{12}$, o caso clínico apresentado buscou aumentar a DVO através das PDP para diminuir o estresse da musculatura acometida pelo bruxismo e consequentemente buscando proteger os elementos dentários de maneira significativa, até que houvesse uma reeducação neuromuscular da região.

Desde muito tempo, Planas ${ }^{24}$ preocupava-se com a etiologia e o diagnóstico de disfunções presentes na oclusão no período da infância, sua ideia era tratar e estimular o desenvolvimento fisiológico do sistema estomatognático e consequente ajuste da DVO através do acréscimo de material, logo, o tratamento criado por ele, conhecido como PDP (pistas diretas planas), consiste no acréscimo de resina fotoativada na superfície oclusal de determinados elementos decíduos, de forma que fiquem planos e estabeleçam o correto posicionamento da mandíbula.

Ainda de acordo com Planas ${ }^{24}$, o tratamento com PDP surgiu para o ajuste de mordida cruzada, sendo utilizada até hoje para essa disfunção, todavia, outros autores como Gribel $^{25}$ enfatizam que sua utilização faz parte da Reabilitação Neuro-Oclusal (RNO), servindo como excelentes alternativas para outras disfunções, já que normaliza não só a oclusão dentária, como também age na postura mandibular, posição dos côndilos nas articulações temporomandibulares e função mastigatória.

O caso clínico apresentado corrobora com os achados de Planas ${ }^{24}$ e Gribel ${ }^{25}$, visto que 0 uso das PDP foram eficazes no tratamento do bruxismo infantil, o infante relatou alívio em sua articulação temporomandibular, a mãe da criança afirmou não ter ouvido o ranger dos dentes do filho durante à noite, além de ter 
percebido uma melhora no seu sono noturno. Simões ${ }^{26}$, Valério et al. ${ }^{27}$ e Garbin et al. ${ }^{28}$ expõem as principais vantagens da instalação de PDP, como sua forma simples de instalação e adaptação, custos mínimos e ação contínua após instalação.

De encontro com Simões ${ }^{26}$ e Valério et al. ${ }^{27}$ o caso apresentado demonstrou que as PDP são fabricadas de maneira simples e prática (uso de resina composta), sem custo elevado e em um serviço público de ensino universitário, além da ausência da necessidade da colaboração do paciente durante 0 tratamento.

CONCLUSÃO

De acordo com o embasamento teórico adquirido, considera-se o bruxismo um hábito parafuncional de etiologia multifatorial, assim como seu tratamento deve ser individualizado. As possíveis consequências dessa disfunção incluem a perda de dimensão vertical, causada pelo desgaste excessivo, trauma de tecidos periodontais, dores na ATM, sensibilidade e perda dentária, apinhamentos, dores de cabeça, aceleração da rizólise de elementos decíduos e perda precoce dos mesmos.

O tratamento escolhido no relato foi a instalação de pistas diretas planas, técnica bastante prática, reversível e que proporciona relaxamento muscular. Deve-se deixar claro que por se tratar de um problema multifatorial não se descarta a possibilidade de encaminhamento para outros profissionais de saúde para melhor acompanhamento.

\section{REFERÊNCIAS}

1. Lipp Men, Souza EAPS, Romano ASF, Colovan MA. Como enfrentar o stress infantil. São Paulo: Ícone; 1991.

2. Kristensen $\mathrm{CH}$, Schaefer LS, Busnello FB. Estratégias de coping e sintomas de stress na adolescência. Estud psicol. 2010;27(1):21-30.

3. Sbaraini CR, Schermann LB. Prevalence of childhood stress and associated factors: a study of schoolchildren in a city in Rio Grande do Sul State, Brazil. Cad Saúde Pública. 2008; 24(5):1082-88.

4. Attanasio, R. Nocturnal bruxism and its clinical management. Dent Clin North Am; 1991;35(1):245-52.

5. Vanderas A.P. Relationship between craniomandibular dysfunction and oral parafunctions in Caucasian children with and without unpleasant life events. J Oral Rehabil; 1995;22:289-94.

6. Loos PJ, Aaron GA. Standards for management of the pediatric patient with acute pain in the temporomandibular joint or muscles of mastication. Pediatr Dent; 1989;11(4):331.
7. Okeson, J. P. Fundamentos de oclusão e desordens têmporo-mandibulares. 2. ed. São Paulo: Artes Médicas; 1992, p.118-135.

8. Feitosa GMA, Félix RCR, Sampaio DC, Vieira ARG, Santos CCO, Fonseca ST. Bruxismo na infância: perfil de comportamento, características do sono e sintomatologia. Rev Bahiana Odontol. 2016;7(2):94-104.

9. Vieira ARG, Drumond CL, Martins PA Jr, Corrêa FP, Gonzaga GC, Marques LS, et al. Prevalence of sleep bruxism and associated factors in preschool children. Pediatr Dent. 2014;36(1):46-50.

10. Serra NJM, Paiva SM, Auad SM, Ramos JML, Pordeus IA. Signs, symptoms, parafunctions and associated factors of parent-reported sleep bruxism in children: a case-control study. Braz Dent J. 2012;23(6):746-52.

11. Castroflorio T, Bargellini A, Rossini G, Cugliari G, Rainoldi A, Deregibus A. Risk factors related to sleep bruxism in children: a systematic literature review. Arch Oral Biol. 2015; 60(11):1618-24.

12. Ahmad, R. Bruxism in children. J Pedodont; 1986, v.10, p.105-125.

13. Maciel, R. N. Oclusão e ATM: procedimentos clínicos. São Paulo: Santos; 1996.

14. Calderan MF, Silva TC, Honório DR, Oliveira TM, Machado MADAM. Fatores etiológicos do bruxismo do sono: revisão de literatura. Rev Odontol Univ Cid São Paulo.2017;26(3):243-49.

15. Halal CSE, Nunes ML. Education in children's sleep hygiene: which approaches are effective? A systematic review. J Pediatr. 2014;90(5): 449-56.

16. Alves VC, Moliterno LF, Ramos ME, Cruz RA, Campos V. Alguns aspectos do bruxismo de interesse do odontopediatra. Rev Odontopediatr; 1993;2:157-63.

17. Hachmann A, Martins EA, Araujo FB, Nunes R. Efficacy of the nocturnal bite plate in the control of bruxism for 3 to 5 year old children. $J$ Clin Pediatr Dent; 1999;24:9-15.

18. Antonio AG, Pierro VS, Maia LC. Bruxism in children: a warning sign for psychological problems. J Can Dent Assoc; 2006;72:155-60.

19.Diniz, M.B., Silva, R.C., Zuanon, A.C.C. Childhood bruxism: a warning sign to pediatric dentists and pediatricians. Rev Paul Pediatr. 2009;27(3):329-34.

20. Gonçalves, L.P.V., Otero, S.A.M., Toledo, O.A. The relationship between bruxism, occlusal factors and oral habits. Dental Press J Orthod; 2010;15(2):97-104.

21. Macedo RC. Bruxismo do sono. R Dental Press Ortodon Ortop Facial; 2008;13(2).

22. Masuko AH, Villa TR, Pradella-Hallinan $M$, et al. Prevalence of bruxism in children with episodic migraine: a case-control study with polysomnography. BMC Res Notes.2014;7: 298. 
23. Paiva T, Batista A, Martins $P$, Martins $A$. The relationship between headaches and sleep disturbances. Headache. 1995;35(10):590-96.

24. Planas, P. Terapia na primeira dentição: verdadeira terapia da "reabilitação neurooclusal", Reabilitação Neuro-oclusal. Rio de Janeiro: Medsi; 1988.

25. Gribel, M.N. Tratamento de mordidas Cruzadas unilaterais posteriores com desvio postural mandibular com Pista Diretas planas. Rev dent press ortodon ortop maxilar. 1999;4(5):47-54.

26. Simões W A. Ortopedia funcional dos maxilares através da reabilitação neuro oclusal. 3. ed. São Paulo: Santos; 2003.

27. Valério, P.; Terçarolli, S.P.; Gribel, M.N. A Ortopedia Funcional na Prevenção de oclusopatias. In: Sakai, E.; Cotrim-Ferreira, F.A.; Martins, N.S. SPO 2012. Nova Visão em Ortodontia e Ortopedia Funcional dos Maxilares. 18. ed. São Paulo: Santos, 2012.

28. Garbin AJI, Wakayama B, Rovida TAS, Garbin CAS. A utilização da Pista Direta de Planas no tratamento precoce da mordida cruzada posterior: relato de caso. Arch Health Invest. 2016;5(4):182-85.

\section{CONFLITO DE INTERESSES}

Os autores declaram não haver conflitos de interesse

\section{AUTOR PARA CORRESPONDÊNCIA}

José Henrique de Araújo Cruz

Rua Paulo Diogenes, 57 - Centro 59990-000 Rafael Fernandes - RN, Brasil

Telefone: (83) 99625-0125.

E-mail: henrique_araujo1992@hotmail.com
Submetido em 28/04/2020

Aceito em 23/10/2020 